\title{
THE INDEPENDENT EFFECT OF SELF-SELECTED VERSUS IMPOSED EXERCISE INTENSITY ON AFFECT
}

\author{
by \\ Luke Haile \\ Bachelor of Science, Bloomsburg University of Pennsylvania, 2005 \\ Master of Science, Bloomsburg University of Pennsylvania, 2008
}

\author{
Submitted to the Graduate Faculty of \\ the School of Education in partial fulfillment \\ of the requirements for the degree of \\ Doctor of Philosophy
}

University of Pittsburgh 


\title{
UNIVERSITY OF PITTSBURGH SCHOOL OF EDUCATION
}

This dissertation was presented

by

\author{
Luke Haile
}

It was defended on

August 10, 2010

and approved by

Elizabeth F. Nagle, Ph. D., Assistant Professor

Joseph L. Andreacci, Ph. D., Associate Professor

Robert J. Robertson, Ph. D., Professor

Dissertation Advisor: Fredric L. Goss, Ph. D., Associate Professor 
Copyright (C) by Luke Haile 2010 


\section{THE INDEPENDENT EFFECT OF SELF-SELECTED VERSUS IMPOSED \\ EXERCISE INTENSITY ON AFFECT}

Luke Haile, Ph. D.

University of Pittsburgh, 2010

Background: The affective response to self-selected and imposed exercise intensities of differing physical stimuli has been previously compared in adults and children. Purpose: The primary purpose of this investigation was to compare the affective response to self-selected (SS) and imposed (IMP) exercise of the same intensity in young, recreationally active adult males. The secondary purpose was to determine if a significant proportion of subjects self-selected exercise intensity above $50 \%$ of oxygen uptake reserve $\left(\mathrm{VO}_{2} \mathrm{R}\right)$. Methods: 32 males [mean(SD)

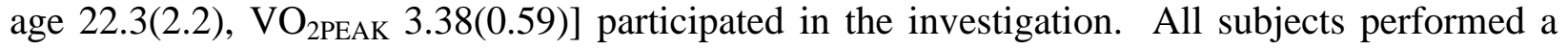
load-incremented $\mathrm{VO}_{2 \text { PEAK }}$ test and a 20 -min, SS exercise trial on a cycle ergometer. One week later, subjects performed the IMP exercise trial. Subjects in the experimental group $(n=16)$ were unaware that the IMP intensity was the same as that previously self-selected. These subjects were told the intensity was 'selected by the investigators.' Control subjects $(\mathrm{n}=16)$ were aware that the intensity of the IMP trial was the same as the SS trial. The affective response measured using Feeling Scale ratings (FS-R) was obtained prior to, during, and following the SS and IMP trials. $\Delta$ FS-R values, calculated by subtracting FS-R estimated during the SS trial from that of the IMP trial at each time point, were analyzed using a mixed-model ANOVA. The proportion of subjects who self-selected intensities above $50 \% \quad \mathrm{VO}_{2} \mathrm{R}$ was tested using a chi-squared analysis. Results: The ANOVA revealed no significant main effects or interaction. The chisquared analysis revealed that a significant $(p<0.05)$ proportion of subjects $(28$ of 33$)$ selfselected exercise intensities above 50\% $\mathrm{VO}_{2} \mathrm{R}$. Conclusions: In the current investigation, the 
affective response to SS and IMP exercise intensities of the same physical stimuli was similar, although there was a considerable amount of inter-individual variability. However, it was found that most subjects self-selected exercise intensities above a level determined by the American College of Sports Medicine to elicit health-fitness benefits. The prescription of SS exercise may be appropriate for young, recreationally active adult males. 


\section{TABLE OF CONTENTS}

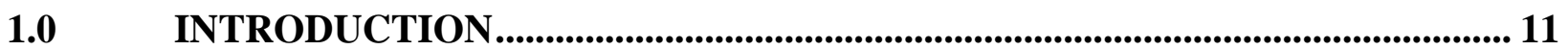

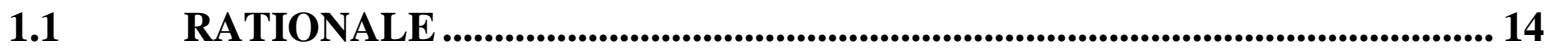

1.2 STATEMENT OF THE PROBLEM .................................................................. 16

1.3 HYPOTHESES ....................................................................................................... 16

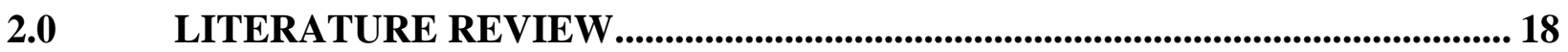

2.1 RATIONALE FOR MEASUREMENT OF AFFECT DURING

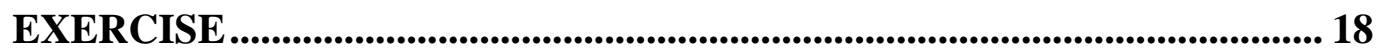

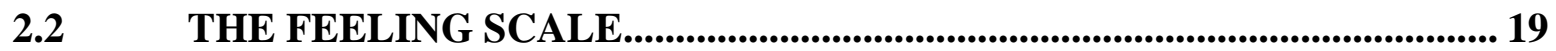

$2.3 \quad$ AFFECT: RELATION WITH EXERCISE INTENSITY .............................. 19

2.3.1 Kirkcaldy and Shephard's Inverted-U Relationship.................................. 19

2.3.2 Ekkakis' Dual-Mode Model................................................................................. 21

$2.4 \quad$ SELF-SELECTED EXERCISE INTENSITY ............................................... 22

2.5 AFFECT DURING SELF-SELECTED VERSUS IMPOSED EXERCISE

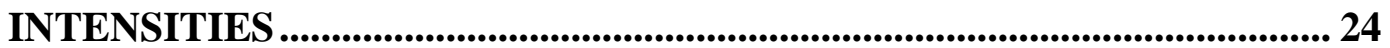

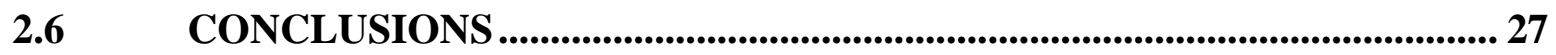

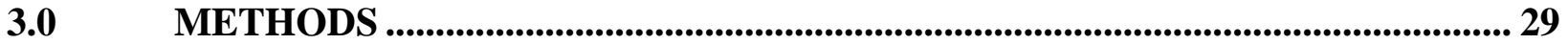

3.1 SUBJECTS ...................................................................................................... 29 
3.2 EXPERIMENTAL DESIGN .

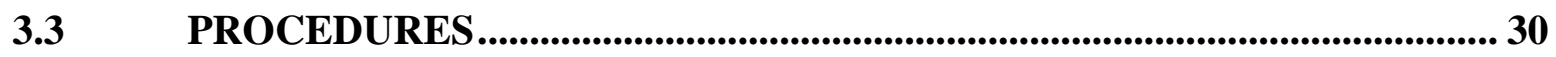

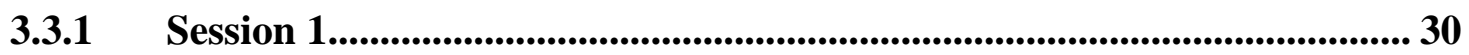

3.3.1.1 Initial Assessments ......................................................................................... 30

3.3.1.2 Peak Oxygen Consumption Test............................................................... 31

3.3.2 Session 2: Self-Selected Exercise Intensity …..................................................... 32

3.3.3 Session 3: Imposed Exercise Intensity ............................................................. 34

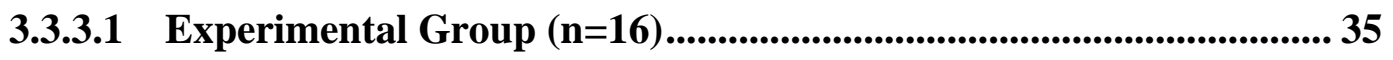

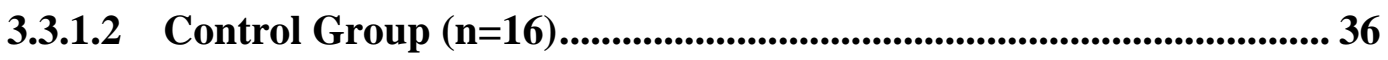

E.4 EXPERIMENTAL VARIABLES ....................................................................... 37

3.4.1 Dependent Variables ....................................................................................... 37

3.4.2 Independent Variable.................................................................................................. 37

3.5 PLANNED STATISTICAL ANALYSES _.......................................................... 37

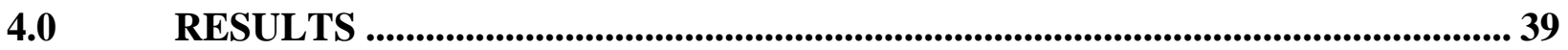

4.1 SUBJECT CHARACTERISTICS _...................................................................... 40

4.2 PRIMARY HYPOTHESIS …...................................................................... 41

4.3 SECONDARY HYPOTHESIS ....................................................................... 44

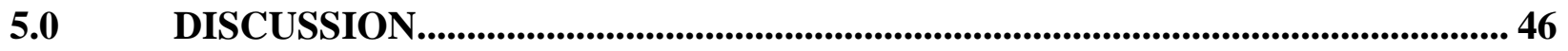

5.1 PRIMARY HYPOTHESIS ............................................................................................ 46

$5.2 \quad$ SECONDARY HYPOTHESIS ................................................................................ 50

$5.3 \quad$ RECOMMENDATIONS FOR FUTURE RESEARCH ...................................5 53

$5.4 \quad$ LIMITATIONS........................................................................................................ 55

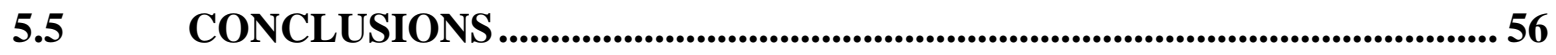


APPENDIX A

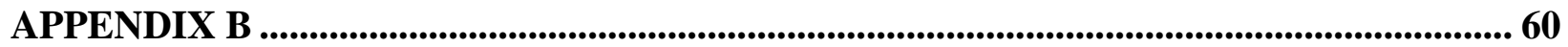

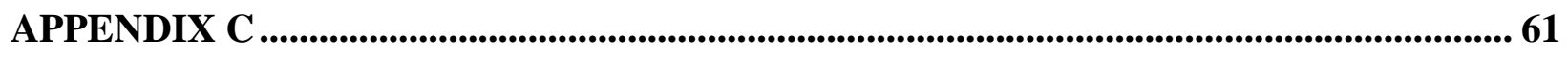

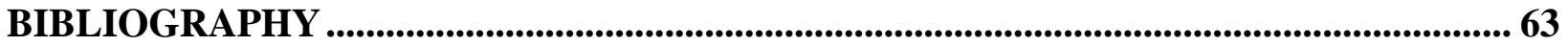




\section{LIST OF TABLES}

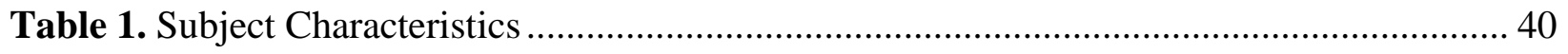

Table 2. Characteristics of Self-Selected Exercise ............................................................ 45 


\section{LIST OF FIGURES}

Figure 1. Difference in Feeling Scale Rating $(\Delta F S-R)$ Between the Self-Selected and Imposed

Exercise Intensity Trials for the Control and Experimental Groups $(p>0.05) \ldots \ldots \ldots \ldots \ldots \ldots \ldots \ldots . . . . . . . . . . . .42$

Figure 2. Control Group Feeling Scale Ratings for the Self-Selected and Imposed Exercise

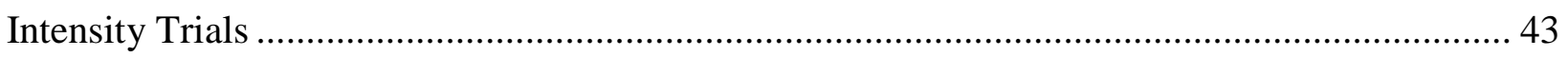

Figure 3. Experimental Group Feeling Scale Ratings for the Self-Selected and Imposed Exercise

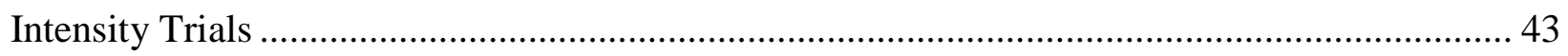




\subsection{INTRODUCTION}

The United States is experiencing a physical inactivity epidemic. As a sedentary lifestyle has been linked to a multitude of cardiovascular and metabolic diseases (Laaksonen et al., 2002; Prasad \& Das, 2009; Venables \& Jeukendrup, 2009), it is a major public health concern that $60 \%$ of adults in the U.S. are physically inactive (Department of Health and Human Services, 1999). In addition, $60 \%$ of individuals who adopt an exercise program drop out within the first 6 months (Pate et al., 1995). Therefore, it is important for health-fitness researchers and professionals to develop effective exercise programs in which unfit or unhealthy individuals are able and willing to participate. Research must be performed to examine the characteristics of exercise programs that deter or facilitate the adoption and maintenance of regular physical activity.

The common strategy within the exercise and health-fitness domain is to prescribe exercise programs similar to the prescription of drugs to treat an ailment. The prescribed exercise intensity is based on scientific evidence showing what will elicit health-fitness benefits for the general population (American College of Sports Medicine, 2006). Therefore, in a typical exercise prescription the intensity is imposed on the individual who is expected to follow the recommendation in order to achieve these benefits. Traditionally, the primary focus of the exercise prescription was to provide a safe and effective program of physical activity. Little or no attention was paid to the emotions or mood experienced by the individual. Since the selfselection of exercise intensity is often absent, this environment can be seen as highly controlling. 
Ekkekakis and Lind (2006) observed that in most studies that have reported low adherence rates, the exercise was prescribed or imposed on the individuals, which may be a primary factor leading to noncompliance. The goal of an exercise prescription should be broadened to include not only the safe completion of each session at an appropriate intensity, but also to facilitate the development of the desire and willingness to continue to participate in exercise in the future, maintain overall healthy behaviors, and ultimately achieve the desired health-fitness benefits.

One strategy to advance the understanding of exercise adherence is to study the acute psychological responses to exercise (Steptoe \& Bolton, 1988). Intrinsic motivation has been identified as an important determinant of exercise maintenance (Omar \& McAuley, 1993). According to the theory of the innate need of self-determination, the controlling manner of prescribing imposed exercise intensities is representative of the type of environment that would not serve to mediate the intrinsic motivation to exercise. Intrinsic motivation is enhanced by the existence of an internal locus of causality which is fostered when one perceives that he or she has a choice over his or her own actions (Deci \& Ryan, 1985). Having more choice in an exercise prescription would serve to satisfy the innate need of self-determination. This could foster the intrinsic motivation which may be the necessary link between an individual's adoption and maintenance of an exercise program (Deci \& Ryan, 1985).

The potential solution to this dilemma is to allow subjects to self-select exercise intensity, thereby fostering an internal locus of causality leading to a greater perception of control over their exercise programs. This internal locus of causality has been proposed to lead to greater enjoyment associated with exercise (Wankel, 1993). In addition, a link has been shown between enjoyment, as well as other intrinsic motives, and adherence (Ryan \& Deci, 2000; Ryan et al., 1997; Caserta \& Gillett, 1998). Enjoyment may be a critical element in promoting exercise 
adherence and improving psychological well-being (Wankel, 1993). According to the theory of planned behavior, the affective component of attitude, which would include the enjoyment of exercise, can be influenced by the nature of the affective responses experienced during individual exercise sessions, whether they are positive or negative. The perceived pleasure or displeasure experienced during exercise could then influence the desire to remain physically active and the willingness to participate in future exercise sessions. Research has shown a link between the affective component of attitude and exercise participation (Godin, 1987; Godin et al., 1995). The affective response to exercise has been proposed as a potential motivational mediator (Ekkekakis et al., 2004). In its most basic context, affect is used to characterize the subjective experience of a valenced state (positive or negative) within a given situation (Ekkekakis \& Petruzzello, 2002). In the context of exercise, affect is used to characterize the general state of each subject, including but not limited to the individual's emotions and mood, specific to the exercise situation. This includes affect prior to, during, and following exercise.

The intensity of exercise has been found to be negatively related to adherence (Cox et al., 2003; Lee et al., 1996; Perri et al., 2002). Individuals generally tend to prefer to perform activities that make them feel good rather than bad (Emmons \& Diener, 1986) and decreased adherence could be due to exercise that is felt as unpleasant because the intensity is above that which is preferred by the individual (Ekkekakis \& Petruzzello, 1999). It has been proposed that moderate intensity exercise elicits the most optimal affective response (Kirkcaldy \& Shephard, 1990), and research has provided evidence to support this hypothesis (Moses et al., 1989). However, other investigations have shown that low or high intensity exercise may also produce positive affect (Ekkekakis et al., 2000; Tate \& Petruzzello, 1995). Therefore, the exercise intensity that induces positive affect can vary between individuals. 
It has been shown that the amount of time spent during a given situation can depend on the affect experienced during the activity (Emmons \& Diener, 1986). Therefore, the acute affective response to exercise may influence future exercise participation. Exercise perceived as feeling pleasant may lead to future participation. On the other hand, exercise perceived as feeling unpleasant could decrease future participation or lead to withdrawal from the activity altogether (Parfitt et al., 2006). The goal, then, would be to maximize the positive affective response that patients or clients experience during exercise, which may be an important link in the chain between exercise and adherence (Van Lunduyt et al., 2000).

\subsection{RATIONALE}

To maximize the energy expenditure of a given exercise session within the time constraints deemed acceptable by an individual, the intensity of exercise must be as high as possible without adverse consequences (Ekkekakis \& Lind, 2006). One primary adverse consequence of which health professionals must be aware is the failure to maintain or even adopt an exercise program. It has been proposed that a causal chain exists, linking a) the intensity of physical activity (not only its level but whether it is self-selected or imposed), b) affective responses (pleasurable versus unpleasurable) and c) adherence. Ekkekakis and Lind (2006) summarized the comparison between allowing individuals to self-select their preferred exercise intensity and prescribing, or imposing, exercise intensity. They stated that when exercise is self-selected, a sense of control is maintained such that the individual can avoid physical discomfort and fatigue. Therefore, selfselected exercise intensity may seem like a somewhat innocuous concept, an emotion such as social physique anxiety may not manifest itself, and the exercise may lead to a positive affective 
response. In contrast, when exercise intensity is imposed, control is taken away and overt signs of fatigue and discomfort may not be avoided. The imposition of exercise intensity may be seen as posing a potential evaluative threat and social physique anxiety may manifest itself leading to decreased pleasure. Consequently, the exercise would lead to a decreased or negative affective response (Ekkekakis \& Lind, 2006). Studies have shown that imposed exercise intensities elicit a more negative (or less positive) affect when compared to self-selected exercise intensities (Ekkekakis \& Lind, 2006; Lind et al., 2008; Parfitt et al., 2000; Parfitt et al., 2006; Rose \& Parfitt, 2007). However, this research has not investigated the independent effect of self-selected versus imposed exercise intensity on affect because the intensities were, in fact, different.

Studies have observed a differential impact of exercise intensity on the affective response (Lind et al., 2008; Parfitt et al., 2006; Parfitt et al., 2000). It has been shown that when individuals are allowed to self-select exercise intensity they tend to choose an intensity that is near the anaerobic threshold or onset of blood lactate accumulation, but may not exceed that level (Dishman et al., 1994; Ekkekakis \& Lind, 2006; Lind et al., 2005). In addition, many adults will self-select exercise intensities within the American College of Sports Medicine's guidelines that will elicit health-fitness benefits (American College of Sports Medicine, 2006; Glass \& Chvala, 2001; Parfitt et al., 2000; Parfitt et al., 2006). However, when exercise intensity is above the anaerobic threshold or onset of blood lactate accumulation, the affective response to exercise systematically decreases, or becomes less pleasurable, in most individuals (Acevedo et al., 2003; Bixby et al., 2001; Ekkekakis et al., 2004; Hall et al., 2002). Therefore, it is important to determine what intensity subjects will self-select so it is known whether self-selected exercise will not only maximize the affect experienced during exercise, leading to future participation, but 
will provide the individual with the necessary exercise stimulus for the achievement of healthfitness benefits.

\subsection{STATEMENT OF THE PROBLEM}

The primary purpose of this investigation was to study the independent effect of self-selected versus imposed exercise intensity on the affective response. The secondary purpose of this investigation was to determine whether the exercise intensity self-selected by the subjects is greater than $50 \%$ of oxygen uptake reserve, the lower limit of the range recommended by the American College of Sports Medicine to achieve health-fitness benefits.

\subsection{HYPOTHESES}

It was hypothesized that subjects in the experimental group would experience less pleasure (or more displeasure) when performing the imposed exercise condition in comparison to the selfselected exercise condition when compared to the control group as evidenced by a significantly lower difference in Feeling scale rating $(\Delta \mathrm{FS}-\mathrm{R})$. This result would indicate a greater decrease in FS-R from the self-selected exercise condition to the imposed exercise condition in experimental subjects compared to control subjects.

It was also hypothesized that subjects would self-select an exercise intensity within American College of Sports Medicine guidelines that have been shown to elicit health-fitness 
benefits, as evidenced by achieving oxygen consumption values averaging greater than $50 \%$ of oxygen uptake reserve. 


\subsection{LITERATURE REVIEW}

\subsection{RATIONALE FOR MEASUREMENT OF AFFECT DURING EXERCISE}

Since the development of the Borg rating of perceived exertion (RPE) scale almost 50 years ago (Borg, 1962), RPE has dominated the literature attempting to explain the subjective response to the exercise stimulus. The Borg 6-20 scale and Robertson's 0-10 OMNI RPE scale (Robertson et al., 2004) have been used to predict peak aerobic capacity and prescribe self-regulated exercise in a wide range of activities and subject populations (Davies et al., 2008; Eston et al., 2008; Goosey-Tolfrey et al., 2010; Kang et al., 2003; Kang et al., 2009). However, Hardy and Rejeski (1989) suggest that since "RPE represents a 'gestalt' of various sensations related to the stress and strain of physical work, it may not accurately reflect a person's mood during exercise." For example, two individuals performing exercise may estimate a RPE of 15 on the Borg scale, however one individual may feel "good" during exercise while the other feels "bad" (Hardy \& Rejeski, 1989). Research has suggested that emotions such as enjoyment, social physique anxiety and the general mood one experiences during exercise can play an important role in the adoption of participation in physical activity, the adherence to an exercise program, and the potential of an individual to withdraw from participating in exercise (Ekkekakis \& Lind, 2006; Parfitt et al., 2006; Wankel, 1993). Therefore, it is not only important to determine "what" one is feeling during exercise, as with RPE, but "how" one feels during exercise. 


\subsection{THE FEELING SCALE}

To answer this question Rejeski and colleagues (1987) developed the Feeling Scale to measure affective responses during exercise. The scale is not designed to measure various categories of emotion. Rather, the Feeling Scale is used to differentiate between feelings along the continuum of core emotions: pleasure or pleasantness versus displeasure or unpleasantness (Frijda, 1988) related to the exercise experience. The Feeling Scale is an 11-point bipolar metric with numbers ranging from -5 to 5. Verbal descriptors situated at each odd integer range from 'Very bad' (at 5), representing maximal displeasure, to 'Neutral' (at 0), to 'Very good' (at 5) representing maximal pleasure (Hardy \& Rejeski, 1989). Since its development, the Feeling Scale has been widely used to measure the affective response of various subject populations during a variety of exercise situations (Ekkekakis et al., 2004; Ekkekakis et al., 2000; Ekkekakis \& Petruzzello, 2002; Lind et al., 2008; Parfitt et al., 2006). In addition, previous research has found the Feeling Scale to be significantly correlated with other self-reported measures of pleasure (Hardy \& Rejeski, 1989). Please see Appendix 1 and 2 to view the Feeling Scale and instructions modified from Hardy and Rejeski (1989).

\subsection{AFFECT: RELATION WITH EXERCISE INTENSITY}

\subsubsection{Kirkcaldy and Shephard's Inverted-U Relationship}

Exercise intensity has a profound influence on affect, but the relation between exercise intensity and affect is not completely clear. Kirkcaldy and Shephard (1990) proposed that moderate 
intensity exercise produces an optimal affective response. Low exercise intensities may be insufficient to lead to positive changes in affect. High exercise intensities may have detrimental effects on the affective response. This produces an inverted-U relationship, with the affective response beginning, most likely, at a level approximating 0 (neutral) on the Feeling Scale during low-intensity exercise, increasing into the positive zone during moderate-intensity exercise, and then decreasing past the neutral zone into the negative zone during high-intensity exercise. Moses and colleagues (1989) found evidence that supports this relation, such that a moderateintensity exercise program ( $\sim 60 \%$ maximal heart rate) produced an optimal affective response, as evidenced by significant reductions in tension and anxiety, in comparison to the low-intensity ( $<50 \%$ maximal heart rate) and high-intensity (70-75\% maximal heart rate) exercise programs.

However, there is also evidence refuting this relation. Tate and Petruzzello (1995) found that high-intensity exercise programs can lead to positive changes in affect, while Ekkekakis and colleagues (2000) found that low-intensity exercise programs can improve affect. There has also been great variation in affect measured during moderate-intensity exercise. In a study by Van Lunduyt and colleagues (2000), of the participants who estimated affect during moderateintensity exercise, $44.4 \%$ experienced increases in affect, $41.3 \%$ experienced a decrease in affect, and $14.3 \%$ experienced no change in affect. Thus, Parfitt and colleagues (2006) suggested that the statements by researchers (Yeung, 1996) and government agencies (Department of Health and Human Services, 1999; Pate et al., 1995) that have deemed moderate-intensity exercise as the optimal intensity to be prescribed may be premature. Moderate exercise intensity "may not be an optimal intensity for some individuals" (Parfitt et al., 2006) and the rigidity of exercise prescription guidelines (American College of Sports Medicine, 2006), specifically regarding exercise intensity (Ekkekakis et al., 2005), may contribute to poor adherence. The 
individualization of exercise prescriptions using measurements of affect may be the optimal approach to maximize adherence.

\subsubsection{Ekkekakis' Dual-Mode Model}

Ekkekakis (2003) proposed the "dual-mode model" to explain inter-individual differences in affect during exercise of varying intensities. As described by Parfitt and colleagues (2006), the model "is based upon the interplay of relevant cognitive processes and interoceptive cues prior to and following the transition from aerobic to anaerobic metabolism," namely, the ventilatory threshold or lactate threshold. At exercise intensities below the anaerobic threshold, when the energy for metabolism is supported by aerobic means, the acute affective response is primarily influenced by cognitive processes such as appraisal, self-efficacy, and social context. These cognitive processes are shaped by personal experience, individual personality variables, personal goal achievement, etc. All of these factors are unique to the individual (Ekkekakis, 2003). Thus, there may be heterogeneity in the affective responses to exercise below the ventilatory threshold due to the inter-individual differences in interpretation of the exercise (Rose \& Parfitt, 2007). At exercise intensities above the anaerobic threshold, when the energy for metabolism is supported by ever-increasing anaerobic means, the acute affective response is primarily influenced by interoceptive cues from baroreceptors, thermoreceptors and visceroreceptors in the heart and lungs (Rose \& Parfitt, 2007). These physiological cues have been referred to as primary emotions because they bypass the cerebral cortex and do not allow for the potential impact of cognitive processes (Damasio, 1995). Thus, there would be less inter-individual variability in the affective responses to exercise above the ventilatory threshold because they are shaped more 
by physiological cues that threaten harm and less by cognitive processes (Ekkekakis, 2003; Parfitt et al., 2006).

Research has supported the 'dual-mode model' in that the acute affective response to exercise declined once the intensity exceeded the ventilatory threshold (Ekkekakis et al., 2005; Hall et al., 2002). It was shown that, while $47 \%$ of subjects exhibited a decline in affect during 15 minutes of treadmill exercise below the ventilatory threshold, $80 \%$ of subjects exhibited a decline in affect during similar exercise above the ventilatory threshold (Ekkekakis et al., 2005). Similar results were found by Parfitt and colleagues (2006) when subjects performed 20 minutes of treadmill exercise. Affective responses were more positive and stable below the lactate threshold with only $25 \%$ of subjects exhibiting a decline, while affective responses became increasingly more negative above the lactate threshold, with $83 \%$ of subjects exhibiting a decline (Parfitt et al., 2006).

\subsection{SELF-SELECTED EXERCISE INTENSITY}

Parfitt and colleagues (2006) suggested that using a self-selected exercise protocol may decrease the amount of inter-individual variability in the acute affective response to exercise. If subjects are allowed to utilize their own personal cognitive appraisal to select intensity during exercise, then this should result in positive affective responses with little variability between subjects. Rather, the variability will be expressed as the different exercise intensities chosen (Rose \& Parfitt, 2007). Two recent studies utilized 20-minute, self-selected treadmill exercise conditions. Parfitt and colleagues (2006) found that sedentary men chose to exercise at an intensity resulting in lactate levels between 3.72 and $4.34 \mathrm{mmol} / \mathrm{L}$, corresponding to approximately $100 \%$ of the 
lactate threshold. Lind and colleagues (2005) found that sedentary women chose to exercise at an intensity resulting in mean oxygen uptake values at the 15 and 20 minute time points that were not different from the ventilatory threshold. However, there was significant variability between subjects, with oxygen uptake values ranging from 62 to $160 \%$ of the ventilatory threshold (Lind et al., 2005). Dishman and colleagues (1994) studied men of low and high physical activity level during 20 minutes of cycling at a preferred level of exertion. The men self-selected exercise intensities with mean values ranging from approximately 50-60\% of peak oxygen uptake at each 5-minute interval. Both groups exhibited a mean self-selected intensity exceeding $60 \%$ of peak oxygen uptake at the 20 -minute time point. This value exceeds that of the mean ventilatory threshold exhibited by the low and high physical activity groups (51.8 and $57.9 \%$ peak oxygen consumption, respectively) (Dishman et al., 1994). These results were also confirmed by Lind and colleagues (2008), who found that middle-aged women self-selected exercise intensity averaging $98 \%$ of the ventilatory threshold.

In contrast, Ekkekakis and Lind (2006) found that normal-weight and overweight women self-selected exercise intensities consistently below the ventilatory threshold. In addition, Rose and Parfitt (2007) found that sedentary men chose to exercise at an intensity resulting in mean lactate levels between 2.06 and $2.52 \mathrm{mmol} / \mathrm{L}$, which were lower but not significantly different from the lactate threshold. The affective response to exercise was evaluated using the Feeling Scale in the studies by Ekkekakis and Lind (2006), Lind et al. (2005), Parfitt et al. (2006) and Rose and Parfitt (2007), and the self-selected exercise conditions resulted consistently in a positive and stable affective response. Regardless, the average intensity self-selected by subjects in these studies corresponds to that recommended by the American College of Sports Medicine for the development and maintenance of cardiorespiratory fitness (American College of Sports 
Medicine, 2006). However, given that the reported values are group means, it is possible that many individuals do not self-select an exercise intensity at a level that will provide physiological and psychological benefits.

\subsection{AFFECT DURING SELF-SELECTED VERSUS IMPOSED EXERCISE INTENSITIES}

A number of recent studies have compared affect measured using the Feeling Scale during selfselected, or preferred, exercise intensities and imposed, or prescribed, exercise intensities. These paradigms have tested the difference in affect between exercise situations where subjects are allowed to choose their own exercise intensity and those where subjects are told what exercise intensity to perform, such as what normally occurs in the health-fitness setting. Recent studies in sedentary male (Parfitt et al., 2006), female (Ekkekakis \& Lind, 2006; Lind et al., 2008; Rose \& Parfitt, 2007) and overweight female adults (Ekkekakis \& Lind, 2006) have utilized imposed exercise intensities corresponding to a level below the lactate threshold, above the lactate threshold (Parfitt et al., 2006; Rose \& Parfitt, 2007), equal to the lactate threshold (Rose \& Parfitt, 2007), and 10\% higher than the self-selected condition (Ekkekakis \& Lind, 2006; Lind et al., 2008). In addition, a recent study compared a self-selected exercise condition to imposed intensities above and below the ventilatory threshold in young adolescent boys and girls (Sheppard \& Parfitt, 2008).

Ekkekakis and Lind (2006) compared affect during a self-selected exercise intensity to the affect experienced during an intensity $10 \%$ higher than the self-selected condition in normalweight sedentary and overweight women (mean age 43 years). Each subject performed 20 
minutes of treadmill exercise during each condition. The self-selected exercise condition corresponded to an intensity below the ventilatory threshold, while the imposed exercise condition corresponded predominately to an intensity above the ventilatory threshold. In normal-weight and overweight women, there were no differences in Feeling Scale ratings between the self-selected and imposed intensities. In both samples, the exercise conditions elicited mean affective responses between +2 and +3 on the Feeling Scale. In overweight women, affect declined significantly throughout the imposed exercise bout, while remaining stable during the self-selected exercise bout. Affect was significantly different between normalweight and overweight women at the 15-minute time point during the imposed exercise intensity, only, where normal-weight women reported a higher affective response than overweight women (Ekkekakis \& Lind, 2006). The investigators suggested that the $10 \%$ increased intensity in the imposed condition was enough to disable participants from achieving both a physiological and affective steady state (Lind et al., 2008).

Parfitt and colleagues (2006) compared affect during self-selected exercise to the affect experienced during prescribed exercise intensities below and above the lactate threshold in sedentary males (mean age 36.5 years). Each subject performed 20 minutes of treadmill exercise for each condition. There were no differences in affect between the self-selected intensity and the imposed intensity below the lactate threshold, which were performed at estimated oxygen consumption levels of 54.1 and $39.8 \%$ of maximal oxygen uptake, respectively. The intensity that was self-selected by subjects was approximately equal to the lactate threshold. Affect measured during exercise above the lactate threshold declined significantly over time, with the mean value becoming less positive and eventually negative by the 20 -minute time point. In the 
other two conditions affect remained stable and positive, approximating a value of +3 on the Feeling Scale (Parfitt et al., 2006).

Rose and Parfitt (2007) compared affect during self-selected exercise to the affect experienced during imposed exercise intensities set at levels below, above, and equal to the lactate threshold. Sedentary women (mean age 39.4 years) performed 20 minutes of treadmill exercise for each condition. The estimated percent of maximal oxygen consumption was significantly different between all conditions, although the mean blood lactate concentration was similar between the self-selected exercise condition and the intensities set at a level equal to the lactate threshold and below the lactate threshold. The above lactate threshold condition resulted in a mean affective response that declined significantly from the 10 to 15 -minute time points and remained negative throughout exercise with values ranging from -.33 to -1.89 . These values were significantly lower than the affect experienced during the other three conditions, during which the mean affective responses remained stable and positive. The self-selected condition resulted in a significantly more positive affective response compared to the exercise intensity set at a level equal to the lactate threshold, with mean values at 5-minute intervals ranging from +2.44 to +2.78 and +1.00 to +1.28 on the Feeling Scale, respectively. In addition, the imposed intensity below the lactate threshold elicited a similar affective response to the self-selected exercise intensity and the imposed intensity equal to the lactate threshold (Rose \& Parfitt, 2007).

Lind, Ekkekakis, and Vazou (2008) compared affect during self-selected exercise to the affect experienced during an imposed intensity $10 \%$ higher than the self-selected intensity. Sedentary women (mean age 43.7 years) performed 20 minutes of treadmill exercise in each condition. At the 20-minute time point, the average exercise intensities were equal to 98 and $115 \%$ of the ventilatory threshold for the self-selected and imposed intensities, respectively. 
Subjects were able to maintain a stable affective response during the self-selected condition, but Feeling Scale ratings declined significantly during the imposed intensity that was only $10 \%$ higher (Lind et al., 2008).

Sheppard and Parfitt (2008) studied the affective response between self-selected and imposed exercise intensities in young adolescent boys and girls self-reported to be active and moderately fit. The subjects (mean age 13.3 years) performed 15 minutes of cycle ergometer exercise at the self-selected intensity and at imposed intensities below the ventilatory threshold ( $80 \%$ of power output corresponding to the ventilatory threshold) and above the ventilatory threshold (130\% of power output corresponding to the ventilatory threshold). The imposed exercise intensity above the ventilatory threshold resulted in a significantly lower and declining affective response compared to the imposed intensity below the ventilatory threshold and the self-selected condition, which were similar and stable over time. The mean affective response for the imposed intensities above and below the ventilatory threshold were +0.42 and +2.50 , respectively, while the self-selected exercise intensity elicited an affective response of +2.84 on the Feeling Scale (Sheppard \& Parfitt, 2008).

\subsection{CONCLUSIONS}

Research that has compared affect during self-selected and imposed exercise intensities provides promising results for the future of exercise prescription and, hopefully, the adherence to

prescribed exercise programs. It has been shown that many individuals experience similar levels of emotions and mood related to a self-selected exercise intensity as compared to an imposed intensity that is actually lower than the self-selected intensity (Ekkekakis \& Lind, 2006; Lind et 
al., 2008; Parfitt et al., 2006; Rose \& Parfitt, 2007). This indicates that subjects may be willing to perform a higher exercise intensity when they are allowed to self-select their preferred level compared to when exercise intensity is imposed. In addition, many individuals will self-select exercise intensities within the American College of Sports Medicine Guidelines that will elicit cardiorespiratory improvements (Dishman et al., 1994; Lind et al., 2005; Lind et al., 2008; Parfitt et al., 2006; Rose \& Parfitt, 2007). Therefore, the prescription of self-selected exercise may not only maximize affect and result in improved adherence to exercise programs, but may provide the individual with physiological benefit as well.

This evidence is further supported by the results of Rose and Parfitt (2007). In their study of sedentary women, blood lactate concentrations elicited by the self-selected intensity and the imposed intensity equal to the lactate threshold were similar, with resultant affective responses being significantly more positive in the self-selected exercise condition. The better mood experienced during self-selected exercise suggests the subjects would prefer performing exercise in that condition versus performing the exercise in the imposed condition. Since subjects were unaware of the similarity between intensities, these results indicate that there may be an independent effect of self-selected versus imposed exercise intensity on affect. Therefore, research is warranted that that is specifically designed to explore the independent effect of selfselected versus imposed exercise intensity on affect. This could be investigated by employing a deception paradigm in which subjects are unaware that an imposed exercise condition is, in fact, the exact same exercise intensity that they previously self-selected in a different exercise session. 


\subsection{METHODS}

\subsection{SUBJECTS}

33 healthy recreationally active males aged 18 to 30 years were recruited from the university community to participate in this investigation. Subjects were considered recreationally active if they performed no more than 150 minutes and no less than 50 minutes of aerobic activity per week. All participation was voluntary, and each subject completed a Physical Activity Readiness Questionnaire (PAR-Q), Medical History Questionnaire, and informed consent prior to entering the study. The study received approval from the University of Pittsburgh Institutional Review Board prior to data collection. Exclusion criteria included: 1) responding "yes" to one or more questions on the PAR-Q; 2) presence of a serious or unstable medical condition; 3) cardiovascular, musculoskeletal and metabolic contraindications to exercise participation; 4) current treatment for a psychological disorder; 5) knowingly taking any performance enhancing substances; 6) unwilling to perform or participate in any component of the investigation; and 7) performed less than 50 or more than 150 minutes of aerobic activity per week.

\subsection{EXPERIMENTAL DESIGN}

A true experimental design compared the effect of self-selected and imposed exercise intensities

on affect. Subjects were randomly assigned to the experimental or control group. All subjects 
performed the self-selected exercise intensity trial first. Deception was employed so that each subject in the experimental group was unaware that the second condition, the imposed exercise intensity, was in fact the same intensity the subject previously self-selected. Each subject in the control group, for the second condition, performed the same previously self-selected intensity without the employment of deception.

\subsection{PROCEDURES}

\subsubsection{Session 1}

3.3.1.1 Initial Assessments All procedures were conducted in the Center for Exercise and Health-Fitness Research (CEHFR) by the primary investigator. For each individual, the three exercise sessions were performed at the same time of day, plus or minus one hour, to control for changes in mood related to the time of day. Pre-trial instructions included the following: A) no aerobic or resistance exercise performed in the 24-hour period prior to exercise; B) no alcohol consumed in the 48-hour period prior to exercise; C) no food consumed in the 2hour period prior to exercise; D) the subject should have arrived normally hydrated; E) the subject should have arrived under normal conditions of caffeine consumption to control for the potential impact of changes in caffeine consumption on mood.

Following completion of the informed consent and questionnaires, height was assessed using a physician scale (Detecto, Webb City, MO). Weight and percent body fat were assessed using a bioelectric impedance analysis scale (Body Composition Analyzer, Model TBF-300A, 
Tanita, Arlington Heights, IL). Subjects were read instructions on how to use the Feeling Scale to estimate affect (Hardy \& Rejeski, 1989). The Feeling Scale and instructions modified from Hardy and Rejeski (1989) are presented in Appendix A and B. Subjects were also read instructions for use of the 0-10 Adult OMNI-Cycle rating of perceived exertion (RPE) scale (Robertson et al., 2004).

3.3.1.2 Peak Oxygen Consumption Test All exercise testing was performed on an electronically-braked cycle ergometer (Corival, Lode, Groningen, Netherlands). All measurements of total body oxygen consumption $\left(\mathrm{VO}_{2}\right)$ and carbon dioxide production $\left(\mathrm{VCO}_{2}\right)$ were performed using a metabolic measurement system (TrueOne 2400, ParvoMedics, Sandy, UT) calibrated prior to each exercise test using standard calibration gases $\left(16 \% \mathrm{O}_{2}, 4 \% \mathrm{CO}_{2}\right.$, nitrogen balanced). After being seated on the cycle ergometer, the headgear, metabolic mouthpiece and nose clip were fitted on the subject to enable measurement of $\mathrm{VO}_{2}$. In addition, the subject was fitted with a heart rate (HR) monitor (Polar Electro, Kempele, Finland). Prior to exercise, the subject sat quietly on the cycle for 2 minutes so that resting measurements of $\mathrm{VO}_{2}$ and HR could be acquired. Subjects maintained a cycling cadence of 50 revolutions per minute throughout the entire test by following a metronome. Following a 2-minute warm-up with no resistance, the power output (PO) was automatically adjusted to 75 watts (W) and increased by $75 \mathrm{~W}$ every 2 minutes. The subject was instructed to continue cycling as long as possible while maintaining the 50 RPM cadence. The test was terminated when the subject could no longer maintain the cadence owing to physical exhaustion. Heart rate was recorded during the final 15 seconds of each 2-minute stage during the exercise test. In addition, the final 30-second average $\mathrm{VO}_{2}$ of each completed 2-minute exercise stage was recorded. Immediately following 
termination, the mouthpiece and nose clips were removed from the subject. The subject then began a 5-minute cool-down period of pedaling against zero resistance at a self-selected cadence. Following the cool-down period, the subject began 15 minutes of quiet, seated recovery on a chair within the laboratory.

Subjects estimated affect immediately prior to the warm-up, at 1:30 of the warm-up and each exercise test stage, and at 4:30 of the 5-minute cool-down and each 5-minute period following exercise up to and including 15 minutes of seated recovery. Subjects estimated undifferentiated [overall body (RPE-O)] and differentiated [legs (RPE-L); chest/breathing (RPEC)] RPE 20 seconds prior to the end of each 2-minute exercise stage in random, counterbalanced order.

Peak oxygen consumption $\left(\mathrm{VO}_{2 \mathrm{PEAK}} ; 1 \cdot \mathrm{min}^{-1}\right)$ was taken as the highest $\mathrm{VO}_{2}$ measured during any 15-second interval of the exercise test. This assessment was used to describe the fitness level of the subjects. In addition, $\mathrm{VO}_{2 \mathrm{PEAK}}$ was used to calculate the percent of $\mathrm{VO}_{2}$ reserve associated with the exercise intensity performed in Session 2, the self-selected intensity. These data were used to test the secondary hypothesis. This is further explained in Section 3.5.

\subsubsection{Session 2: Self-Selected Exercise Intensity}

At least 48 and no more than 96 hours following Session 1 at approximately the same time of day ( \pm 1 hour) subjects will returned to the CEHFR to perform 20 minutes of submaximal exercise on a cycle ergometer. Prior to beginning exercise, subjects were read the following instructions: "Today, I will ask you to select an intensity that you prefer to perform on the cycle. This should be an intensity that you would choose for a 20 -minute workout if you were 
participating in a fitness program. The intensity should be high enough that you would get a good workout, but not so high that you would not prefer to exercise at that intensity daily or at least every other day. It should be an intensity that is appropriate for you" (Dishman et al., 1994; Parfitt et al., 2000).

In addition, subjects were instructed that they must maintain a 50 RPM cadence throughout the exercise and that they will have the opportunity to adjust the resistance for 1 minute after 5, 10, and 15 minutes during the test. Prior to the test, the display screen on the cycle ergometer was covered so the subject was not aware of the actual PO. Following a 2minute warm-up at $0 \mathrm{~W}$, each subject had 1 minute to select the desired intensity. All adjustments were made by the research technician using the manual control setting on the computer to which the ergometer was interfaced. Initially, the PO was adjusted to $80 \mathrm{~W}$ during the final 10 seconds of the 2-minute warm-up. In 15 second intervals (at 0:00, 0:15, 0:30, 0:45, and 1:00), the technician asked the subject if he would like the intensity to be 'harder', 'easier', or 'the same'. The subject was instructed to respond to the technician using the following hand signals: thumb up for 'harder'; thumb down for 'easier'; and moving the hand from side to side for 'the same'. The initial adjustments requested by the subject were made in $20 \mathrm{~W}$ increments. If the subject requested a change in the direction of PO adjustment, further adjustments were made in $10 \mathrm{~W}$ increments. If the subject requested an additional change in the direction of PO adjustment, further adjustments were made in $5 \mathrm{~W}$ increments until the subject responded with 'the same'.

For example, at the 0:00 time point of the initial one-minute adjustment period the subject signals for the intensity to be made 'harder'. The research technician increases the PO from $80 \mathrm{~W}$ to $100 \mathrm{~W}$. At the 0:15 time point the subject signals for the intensity to be made 
'harder' again. The research technician increases the PO from $100 \mathrm{~W}$ to $120 \mathrm{~W}$. At the $0: 30$ time point the subject asks for the intensity to be made 'easier'. The technician decreases the PO from $120 \mathrm{~W}$ to $110 \mathrm{~W}$. At the $0: 45$ time point the subject signals for the intensity to be made 'harder'. The technician increases the PO from $110 \mathrm{~W}$ to $115 \mathrm{~W}$. At the 1:00 time point of each adjustment period each subject will be asked if he would like to make a final adjustment.

In addition, each subject had 1 minute to adjust the PO after 5, 10, and 15 minutes if desired. The PO was recorded after each adjustment period. $\mathrm{VO}_{2}$ and $\mathrm{HR}$ data were collected continuously throughout the test, similarly to Session 1, and were recorded every 5 minutes. Following the 20-minute exercise period, the mouthpiece and nose clips were removed from the subject. The subject immediately began a 5-minute cool-down period of pedaling against zero resistance at a self-selected cadence. Following the cool-down period, the subject began 15 minutes of quiet, seated recovery on a chair within the laboratory.

Subjects estimated affect immediately prior to the warm-up, at 1:30 of the warm-up, and at 4:30 of each 5-minute exercise period, the 5-minute cool-down, and each 5-minute period following exercise up to and including 15 minutes of seated recovery. Subjects estimated RPEO, RPE-L and RPE-C 20 seconds prior to the end of each 5-minute exercise period in random, counterbalanced order.

\subsubsection{Session 3: Imposed Exercise Intensity}

At least 96 and no more than 192 hours (4-8 days) following Session 2, subjects returned to the CEHFR to perform 20 minutes of submaximal exercise on a cycle ergometer. 
3.3.3.1 Experimental Group $(\mathbf{n}=16)$ Prior to exercise and estimations of affect, subjects were read the following instructions: "you will perform a 20 minute exercise session at a resistance selected by the investigators." Subjects were instructed to maintain a 50 RPM pedal cadence throughout the exercise. Prior to the test, the display screen on the cycle ergometer was covered so the subject was not aware of the actual PO. All adjustments in resistance during Session 3 were pre-programmed into the personal computer interfaced to the cycle ergometer and automatically occurred at the designated time. Following a 2-minute and 30-second warmup at $0 \mathrm{~W}$, the resistance was adjusted to the initial PO selected by the subject during Session 2. This initial adjustment was automatically performed at 0:30. At 5:30, 10:30, and 15:30 during the test, the resistance was adjusted to that previously selected by the subject during the same respective time point during Session 2. These time points bisect the adjustment period allowed for the subject in Session 2. $\mathrm{VO}_{2}$ and $\mathrm{HR}$ data were collected continuously throughout the test, similarly to the previous sessions, and were recorded every 5 minutes. Following the 20-minute exercise period, the mouthpiece and nose clips were removed from the subject. The subject immediately began a 5-minute cool-down period of pedaling against zero resistance at a selfselected cadence. Following the cool-down period, the subject began 15 minutes of quiet, seated recovery on a chair within the laboratory.

Subjects estimated affect immediately prior to the warm-up, at 1:30 of the warm-up, and at 4:30 of each 5-minute exercise period, the 5-minute cool-down, and each 5-minute period following exercise up to and including 15 minutes of seated recovery. Subjects estimated RPEO, RPE-L and RPE-C 20 seconds prior to the end of each 5-minute exercise period in random, counterbalanced order. 
3.3.3.2 Control Group $(\mathbf{n}=\mathbf{1 6}) \quad$ Prior to exercise and estimations of affect, subjects were read the following instructions: "you will perform 20 minutes of exercise using the same resistance that you selected during the previous session." Subjects were instructed to maintain a 50 RPM pedal cadence throughout the exercise and that the resistance will be adjusted by an investigator at 5, 10, and 15 minutes during the test exactly as the subject adjusted the resistance in the previous session. Prior to the test, the display screen on the cycle ergometer was covered so the subject was not aware of the actual PO. Following a 2-minute warm-up at $0 \mathrm{~W}$, the resistance was adjusted to the initial PO selected by the subject during Session 2. This initial adjustment was automatically performed at 0:30. At 5:30, 10:30, and 15:30 during the test, the resistance was adjusted to that previously selected by the subject during the same respective time point during Session 2. $\mathrm{VO}_{2}$ and $\mathrm{HR}$ data were collected continuously throughout the test, similarly to the previous sessions, and were recorded every $5 \mathrm{~min}$. Following the 20 -minute exercise period, the mouthpiece and nose clips were removed from the subject. The subject immediately began a 5-minute cool-down period of pedaling against zero resistance at a selfselected cadence. Following the cool-down period, the subject began 15 minutes of quiet, seated recovery on a chair within the laboratory.

Subjects estimated affect immediately prior to the warm-up, at 1:30 of the warm-up, and at 4:30 of each 5-minute exercise period, the 5-minute cool-down, and each 5-minute period following exercise up to and including 15 minutes of seated recovery. Subjects estimated RPEO, RPE-L and RPE-C 20 seconds prior to the end of each 5-minute exercise period in random, counterbalanced order. 


\subsection{EXPERIMENTAL VARIABLES}

\subsubsection{Dependent Variables}

The dependent variable associated with the primary hypothesis included subject estimations of affect prior to, during, and following exercise in Session 2 and Session 3. The dependent variable used in the test of the secondary hypothesis was oxygen uptake measured during the self-selected exercise intensity trial only, Session 2.

\subsubsection{Independent Variable}

The independent variable was the exercise stimulus with two separate levels: self selected exercise intensity and imposed exercise intensity.

\subsection{PLANNED STATISTICAL ANALYSES}

A power analysis (G*Power 3.0.10) was performed for the primary hypothesis. With a given alpha level of 0.05 , a total sample size of 32 males (16 per group) is necessary to satisfy an effect size of 0.3 with $90 \%$ power. Statistical analyses were performed using SPSS 17.0 for Windows. Subject characteristics were compared between groups using independent samples t-tests. The difference in affect at each of 10 time-points [prior to and during warm-up (2), each 5-minute period during exercise (4), during cool-down (1), each 5-min period during exercise recovery (3)] were calculated for each subject by subtracting the Feeling Scale rating measured at that 
time-point during Session 2 (the self-selected intensity) from the Feeling Scale rating measured at that time-point during Session 3. These values were compared between the experimental and control groups using a 2 (group) by 10 (time) mixed-model analysis of variance (ANOVA) with repeated-measures. The primary objective of this analysis was to test the independent effect of self-selected versus imposed exercise intensity on affect. Statistical significance was accepted at the $p<0.05$ level of confidence. In addition, the final 30-second $\mathrm{VO}_{2}$ measurements taken at the end of each 5-minute period during the self-selected exercise session were averaged for each subject. Chi squared analysis determined if the proportion of subjects that self-selected exercise intensities within American College of Sports Medicine guidelines for $\mathrm{VO}_{2}\left(>50 \% \mathrm{VO}_{2}\right.$ reserve) (American College of Sports Medicine, 2006) was greater than the proportion of subjects that self-selected an intensity that is not consistent with the aforementioned guidelines. This analysis used data from Session 2, the self-selected exercise session, from every subject. 


\subsection{RESULTS}

The primary objective of this investigation was to test the independent effect of self-selected versus imposed exercise intensity on the affective response associated with submaximal cycle

ergometer exercise. The secondary objective of this investigation was to test whether a significant proportion of subjects would self-select an exercise intensity within American College of Sports Medicine Guidelines for the achievement of health-fitness benefits.

32 recreationally active male subjects completed all three experimental exercise trials. One subject did not return to the study after completing the second exercise trial, the selfselected exercise intensity trial, and did not complete the imposed exercise intensity trial. Therefore, this subject is included in the subject characteristics for the total sample $(\mathrm{n}=33)$, but not in the subject characteristics for either the control or experimental group. The independent ttests used to compare subject characteristics between groups did not include this subject. However, because this subject completed the self-selected exercise intensity trial, his data were used in the chi-squared analysis to answer the secondary hypothesis. 


\subsection{SUBJECT CHARACTERISTICS}

Subject characteristics (mean \pm SD) are shown in Table 1 for the total sample and separately for the control and experimental groups. All subject characteristics were similar $(p>0.05)$ between the control and experimental groups.

Table 1. Subject Characteristics.

$\underline{\text { Total }(\mathrm{n}=33)} \quad \underline{\text { Control }(\mathrm{n}=16)} \quad \underline{\text { Experimental }(\mathrm{n}=16)}$

Age (yr)

$22.3 \pm 2.2$

$22.1 \pm 2.3$

$22.6 \pm 2.2$

Height $(\mathrm{cm})$

$180.9 \pm 6.5$

$181.6 \pm 6.2$

$180.5 \pm 7.1$

Weight (kg)

$81.0 \pm 9.4$

$83.4 \pm 9.3$

$78.9 \pm 9.5$

Fat (\%)

$17.0 \pm 4.7$

$17.9 \pm 4.2$

$16.2 \pm 5.4$

$\mathrm{VO}_{2 \text { PEAK }}\left(1 \cdot \mathrm{min}^{-1}\right) \quad 3.38 \pm 0.59$

$3.43 \pm 0.68$

$3.34 \pm 0.53$

$\mathrm{V}_{\mathrm{PT}}\left(1 \cdot \mathrm{min}^{-1}\right)$

$2.20 \pm 0.53$

$2.32 \pm 0.62$

$2.09 \pm 0.32$

$\mathrm{V}_{\mathrm{PT}}\left(\% \mathrm{VO}_{2 \mathrm{PEAK}}\right)$

$63.9 \pm 8.1$

$65.9 \pm 7.3$

$61.9 \pm 8.9$

$50 \% \mathrm{VO}_{2}$ Reserve

$1.49 \pm 0.29$

$1.51 \pm 0.34$

$1.47 \pm 0.25$

$\left(1 \cdot \min ^{-1}\right)$

Values are mean \pm SD. $\mathrm{V}_{\mathrm{PT}}$ : ventilatory breakpoint. 


\subsection{PRIMARY HYPOTHESIS}

A 2 (group) by 10 (time) mixed-model ANOVA was used to compare the difference in affect from the self-selected exercise intensity trial to the imposed exercise intensity trial between the control and experimental groups across time. The ANOVA tested main effects for group and time, as well as the interaction effect. The ANOVA tables for within and between subjects effects can be found in Appendix C.

The main effect for group was not significant, $F(1,30)=0.277, p=0.602$, partial eta squared $=0.009$. The control and experimental groups displayed a similar difference in Feeling Scale rating from the self-selected exercise intensity trial to the imposed exercise intensity trial. The main effect for time was not significant, $F(1,30)=1.325, p=0.224$, partial eta squared $=$ 0.042. Subjects displayed a similar difference in Feeling Scale rating across time. In addition, the group by time interaction was not significant, $F(1,30)=0.964, p=0.470$, partial eta squared $=0.031$. The pattern of change in the difference in Feeling Scale rating from the self-selected exercise intensity trial to the imposed exercise intensity trial was similar between groups and across time points. Figure 1 displays the mean $( \pm \mathrm{SD})$ difference in Feeling Scale rating $(\Delta \mathrm{FS}-\mathrm{R})$ at each time point between the self-selected and imposed exercise intensity trials for the control and experimental groups. These values were used to test the primary hypothesis. 


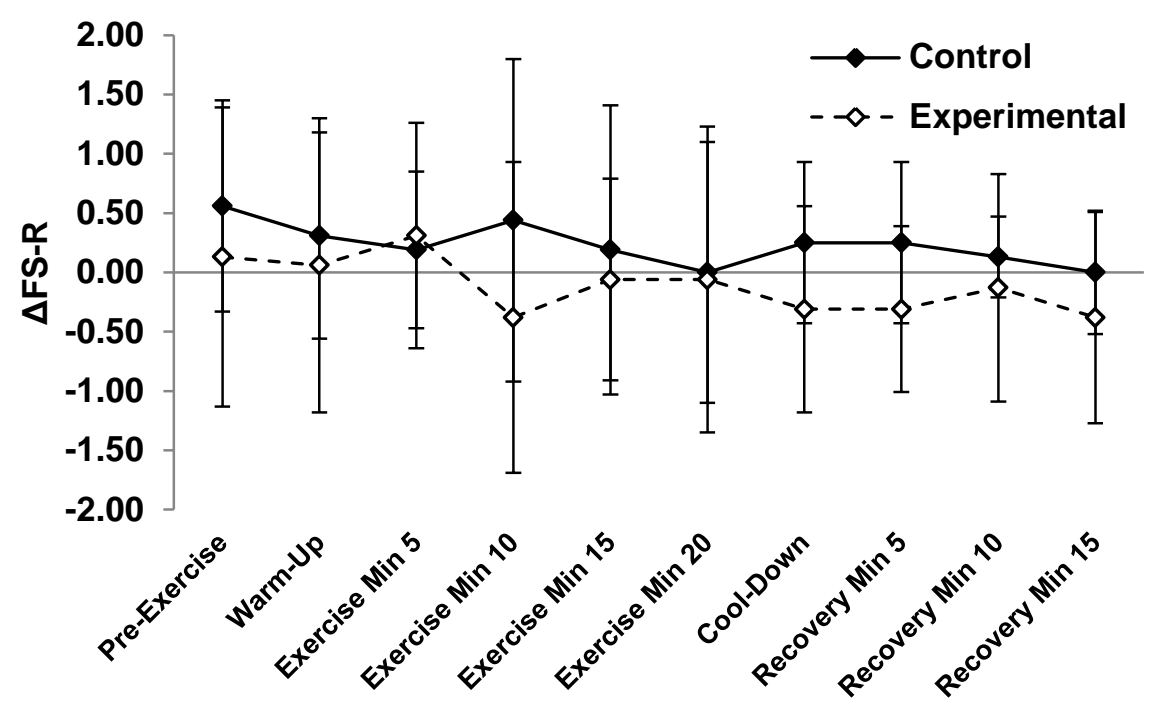

Figure 1. Difference in Feeling Scale Rating ( $\Delta$ FS-R) Between the Self-Selected and Imposed Exercise Intensity Trials for the Control and Experimental Groups $(\boldsymbol{p}>\mathbf{0 . 0 5})$

Figures 2 and 3 display the group means $( \pm$ SD) of the Feeling Scale ratings estimated during the self-selected and imposed exercise intensity trials separately for the control and experimental groups, respectively. These values were those used for the calculation of the $\Delta \mathrm{FS}$ $\mathrm{R}$ values for each group that were used to test the primary hypothesis. 


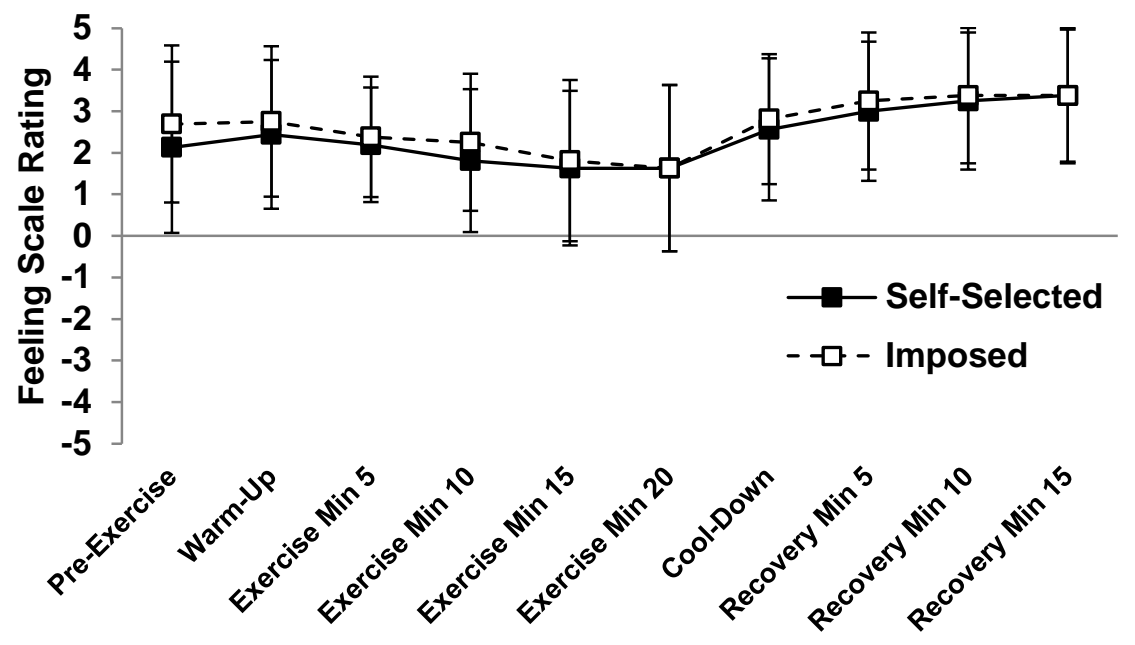

Figure 2. Control Group Feeling Scale Ratings for the Self-Selected and Imposed Exercise Intensity Trials

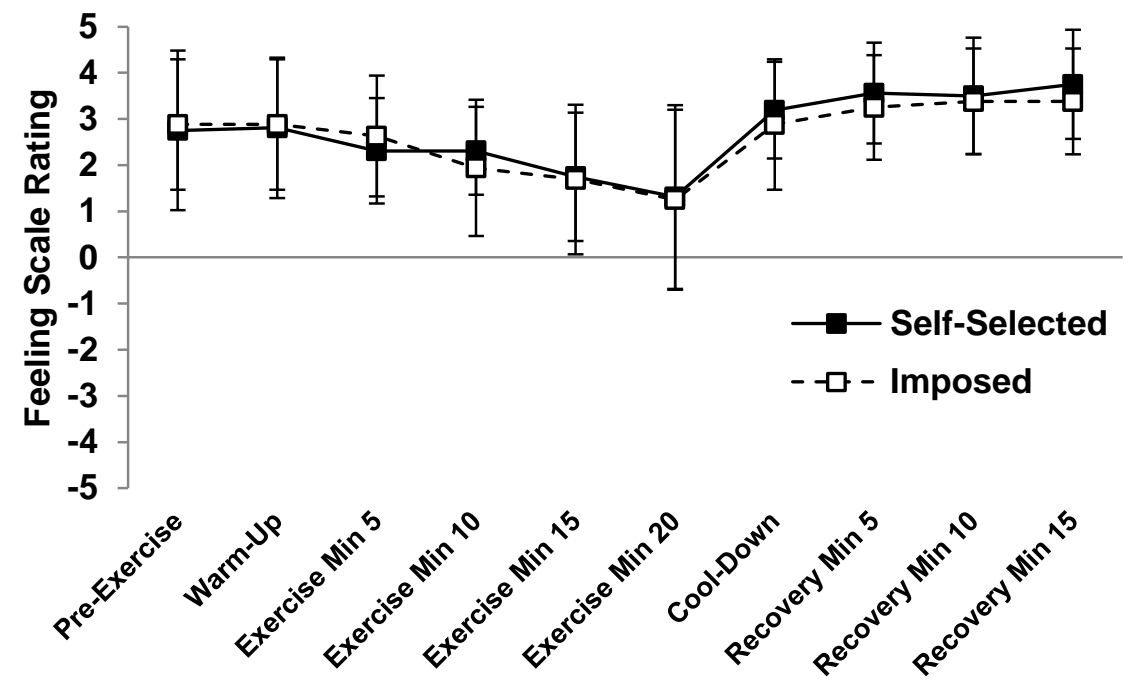

Figure 3. Experimental Group Feeling Scale Ratings for the Self-Selected and Imposed Exercise Intensity Trials 


\subsection{SECONDARY HYPOTHESIS}

A chi-squared analysis was used to determine if a significant proportion of subjects self-selected an exercise intensity above $50 \%$ of $\mathrm{VO}_{2}$ reserve. Twenty-eight of $33(85 \%)$ subjects selfselected an exercise intensity above $50 \%$ of $\mathrm{VO}_{2}$ reserve. This observed proportion is significantly greater than the expected, chi-squared $(1)=16.03, p<0.05$. Table 2 displays the characteristics of the self-selected exercise session for the total sample, subjects who selfselected an exercise intensity above $50 \%$ of $\mathrm{VO}_{2}$ reserve, as well as those who self-selected an exercise intensity below $50 \%$ of $\mathrm{VO}_{2}$ reserve. 
Table 2. Characteristics of Self-Selected Exercise.

\begin{tabular}{|c|c|c|c|}
\hline & $\underline{\text { Total }(n=33)}$ & Above $(n=28)$ & $\underline{\text { Below }(n=5)}$ \\
\hline \multicolumn{4}{|l|}{$\left(1 \cdot \min ^{-1}\right)$} \\
\hline $\mathrm{SS} \mathrm{VO}_{2}\left(1 \cdot \mathrm{min}^{-1}\right)$ & $1.93 \pm 0.47$ & $2.02 \pm 0.45$ & $1.43 \pm 0.14$ \\
\hline $\mathrm{SS} \mathrm{VO}_{2}\left(\% \mathrm{VO}_{2 \mathrm{PEAK}}\right)$ & $57.6 \pm 11.1$ & $60.5 \pm 9.2$ & $40.9 \pm 3.8$ \\
\hline 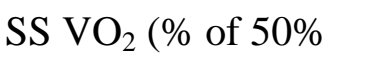 & $130.9 \pm 26.3$ & $137.7 \pm 22.3$ & $93.2 \pm 9.3$ \\
\hline \multicolumn{4}{|l|}{$\mathrm{VO}_{2}$ reserve) } \\
\hline SS power output (W) & $118.8 \pm 33.2$ & $125.0 \pm 32.1$ & $84.0 \pm 10.8$ \\
\hline SS FS-R & $1.8 \pm 1.4$ & $1.7 \pm 1.4$ & $2.1 \pm 1.0$ \\
\hline
\end{tabular}




\subsection{DISCUSSION}

\subsection{PRIMARY HYPOTHESIS}

The primary purpose of this investigation was to test the independent effect of self-selected versus imposed exercise intensity on the affective response. This was performed using a deception paradigm in which a 20 minute exercise session that was identical in physical stimulus to that performed in a self-selected exercise condition one week previous was imposed on subjects in the experimental group. The experimental subjects were told that the intensity was 'selected by the investigators,' thereby creating the imposed exercise environment. These experimental subjects were compared to control subjects who performed the same exercise protocol with knowledge that the intensity performed in the second submaximal exercise session was identical to that previously self-selected. The difference in affect between the self-selected and imposed exercise conditions was calculated by subtracting the Feeling Scale ratings (FS-R) estimated during the self-selected exercise intensity trial from the FS-R estimated during the imposed exercise intensity trial for each time point. This procedure resulted in a set of delta values $(\triangle \mathrm{FS}-\mathrm{R})$ for each subject with a single $\triangle \mathrm{FS}-\mathrm{R}$ representing each individual time point. The $\Delta$ FS-R values calculated for the control group represent the natural week-to-week variability in affect during the performance of the same submaximal cycle ergometer exercise session. The comparison of these values to those of the experimental group sought to determine whether the 
imposition of the same set of exercise intensities (as subjects were allowed to adjust the intensity in 5-minute intervals) previously self-selected would result in a change in FS-R that was different than the change in FS-R that occurred in a similar sample of young, recreationally active adult males in the control condition. Subject characteristics did not differ between the control and experimental groups (Table 1).

It was hypothesized that subjects in the experimental group would experience less pleasure (or more displeasure) when performing the imposed exercise condition in comparison to the self-selected exercise condition when compared to the control group as evidenced by significantly lower, and potentially negative, $\Delta \mathrm{FS}-\mathrm{R}$ values. For example, if a subject in the experimental group estimated a FS-R value of +1 at the 15 -minute time point during exercise in the self-selected exercise trial and, one week later, estimated a FS-R value of -1 at the 15minute time point during exercise in the imposed exercise trial, the $\Delta \mathrm{FS}-\mathrm{R}$ value for the 15 minute time point during exercise would be calculated as -2 . This result would indicate a decrease in FS-R from a self-selected exercise condition to an imposed exercise condition using identical exercise intensities. However, the ANOVA indicated a non-significant main effect for group, such that the control and experimental groups displayed similar $\Delta \mathrm{FS}-\mathrm{R}$ values. Therefore, in the current population of recreationally active, young adult males, there was no difference in $\Delta$ FS-R calculated using FS-R values estimated during the self-selected and imposed exercise conditions between the control and experimental groups. The week-to-week variability in affect measured during the performance of a submaximal cycle ergometer exercise session was similar to the change in FS-R that occurred when the same exercise intensity was imposed on a similar group of individuals who were deceived such that they were not aware the intensity was the same as that previously self-selected. 
In addition, there was a non-significant main effect for time and the group by time interaction. Therefore, $\Delta \mathrm{FS}-\mathrm{R}$ values were similar at each time point and the pattern of change of $\Delta$ FS-R was similar for each group across time. It can be seen in Figure 1 that there was a considerable amount of variability in $\Delta \mathrm{FS}-\mathrm{R}$ at each time point for each group. The standard deviation from the mean $\Delta$ FS-R often exceeded the mean value itself. Some individuals in both groups experienced almost no change in FS-R values whereas others experienced changes up to four FS-R units from the previous week. It is possible that some individuals allow nonphysiological mediators to dominate their reasoning in the estimation of affect, whereas the affective response may be more closely linked to the physical stimulus and the resultant physiological response in other individuals. Because there is such considerable inter-individual variability in the change of FS-R values from week-to-week, future qualitative research should explore the underlying psychosocial factors that may influence the affective response associated with exercise. It is especially important to explore these factors in sedentary individuals who display a certain psychological resistance to the adoption and maintenance of regular physical activity.

It is difficult to compare the current investigation to previous comparisons between selfselected and imposed exercise intensities because this investigation used a true experimental design with randomization to a control and experimental group. In addition, the current investigation calculated delta scores $(\Delta \mathrm{FS}-\mathrm{R})$ by subtracting FS-R collected during the selfselected exercise session from FS-R collected during the imposed exercise session. In contrast, previous investigations utilized quasi-experimental designs in which subjects performed the selfselected and imposed conditions that were compared using analyses with repeated-measures. 
Previous investigations involving comparisons between self-selected and imposed exercise conditions compared FS-R measured during exercise sessions of differing intensity. For example, previous investigations compared a self-selected exercise condition to an intensity $10 \%$ higher than that self-selected (Ekkekakis \& Lind, 2006; Lind et al., 2008) or to intensities set at a certain level below (Parfitt et al., 2006; Rose \& Parfitt, 2007), equal to (Rose \& Parfitt, 2007), or above the lactate threshold (Parfitt et al., 2006; Rose \& Parfitt, 2007). Although recent investigations have found that some individuals will self-select an exercise intensity similar to the lactate threshold (Parfitt et al., 2006) or the ventilatory threshold (Lind et al., 2005), there is such considerable variability from subject to subject that imposing an exercise intensity at a level associated with the lactate or ventilatory threshold will most likely be different from the selfselected exercise intensity in most individuals. For example, Lind et al. (2005) found that subjects self-selected exercise intensities ranging from $62 \%$ to $160 \%$ of the ventilatory threshold, resulting in a mean intensity that was similar to the mean ventilatory threshold. The current investigation found that subjects self-selected exercise intensities ranging from $57 \%$ to $133 \%$ of the ventilatory threshold. Therefore, the best way to compare a self-selected and imposed exercise intensity that is exactly the same is to employ deception and create an environment involving the imposition of the same exercise intensity that was previously self-selected by the subject.

This considerable inter-individual variability in intensity of exercise that was selfselected may have led to a decrease in the influence of the artificial environment of imposed exercise, especially in those individuals who self-selected exercise intensities below the ventilatory threshold and were randomized to the experimental group. It was hypothesized that these subjects would experience a decrease in affect from the self-selected to the imposed 
exercise condition. However, with the imposed exercise condition being the same intensity as that previously self-selected and the subjects most likely assuming that the intensity "selected by the investigators" would be at least more difficult than the intensity they self-selected and possibly even quite difficult, these subjects may have been surprised by the ease of performance of the imposed exercise session. This may have been a potential reason for the minimal change in FS-R between exercise conditions in the experimental group. It was also assumed that the control group would experience similar affect between exercise sessions, which did occur.

\subsection{SECONDARY HYPOTHESIS}

The secondary purpose of this investigation was to determine if young, recreationally active adult male subjects would self-select an exercise intensity that would induce physiological benefits. It was hypothesized that subjects would self-select exercise intensities within American College of Sports Medicine guidelines that have been shown to elicit health-fitness benefits, as evidenced by achieving oxygen consumption values averaging greater than $50 \%$ of oxygen uptake reserve $\left(\mathrm{VO}_{2} \mathrm{R}\right)$. This hypothesis was tested using a chi squared analysis. It was found that a significant proportion of subjects (85\%) self-selected intensities above this cut-point. Only 5 of 33 subjects did not self-select above the expected level. The mean of the self-selected intensities was equal to $65 \% \mathrm{VO}_{2} \mathrm{R}$, which is $131 \%$ of the cut-point, $50 \% \mathrm{VO}_{2} \mathrm{R}$. This value was equivalent to $58 \%$ of $\mathrm{VO}_{2 \mathrm{PEAK}}$ and was associated with a mean power output of 119 watts.

Previous investigations that have studied self-selected exercise intensity have found that many subjects will choose intensities at a level known to elicit health-fitness benefits. However, 
most investigations have described the chosen intensities in relation to indices of the gasexchange threshold, such as the lactate threshold (Parfitt et al., 2006) or ventilatory threshold (Dishman et al., 1994; Lind et al., 2005; Lind et al., 2008). In addition, these investigations have studied older, sedentary and overweight individuals. This is the first known study of the affective response to self-selected exercise that has been conducted using college-aged males.

The exercise intensities self-selected by the subjects in this investigation were associated with exercise estimations of affect with a mean value of +1.8 on the Feeling Scale. Although some individuals did exhibit values above +2 , the mean result is lower than that previously found by Ekkekakis and Lind (2006) and Rose and Parfitt (2007), in which subjects consistently estimated FS-R between +2 and +3 , as well as that previously found by Parfitt and colleagues (2006), in which the mean FS-R during self-selected exercise approximated +3 . It is possible that the young males in the current investigation self-selected exercise intensities that yielded lower FS-R than previous studies because of their perception of exercise that is supposed to be a 'good workout' as stated in the Feeling Scale instruction set adapted from that of Dishman and colleagues (1994). Previous investigations (Ekkekakis \& Lind, 2006; Parfitt et al., 2006) used instructions modified from those of Dishman et al. (1994) such that they removed the phrase 'good workout.' These instructions were first modified for an investigation conducted by Parfitt and colleagues in 2000 that did not employ the Feeling Scale. For example, the instructions used in the investigation conducted by Parfitt and colleagues (2006) used the following instructions for the self-selected exercise session: 'select an intensity that you prefer that can be sustained for 20 minutes and that you would feel happy to do regularly' (Parfitt et al., 2006). For the current investigation, the original instructions described by Dishman were thought to be appropriate for use with a sample of young, recreationally active males. Further research comparing younger and 
older individuals including a qualitative approach and using the same instructions for selfselected exercise may provide information about whether age influences the underlying psychosocial factors associated with exercise undertaken to enhance fitness.

Ekkekakis and Lind (2006) suggest that, in overweight and chronically sedentary populations, emphasis should be placed on self-selected exercise prescriptions rather than the imposition of certain exercise intensities. Overweight women self-selected exercise intensities averaging $69-70 \%$ of peak functional capacity and $85-87 \%$ of peak heart rate, which are clearly strenuous and well within American College of Sports Medicine (ACSM) guidelines for the adequate management of body weight (55-69\% of maximal heart rate) (Ekkekakis \& Lind, 2006). These results are in agreement with those of the current investigation in which a significant proportion of subjects self-selected exercise intensities above $50 \%$ of oxygen uptake reserve $\left(\right.$ mean $=58 \%$ of $\mathrm{VO}_{2 \mathrm{PEAK}}$, which is consistent with ACSM guidelines for exercise undertaken to enhance cardiorespiratory fitness. In both studies, self-selected exercise resulted in a positive affective and physiological response that has previously been shown to produce cardiorespiratory benefits. These data suggest that self-selected exercise intensity can be incorporated into fitness programming in young, recreationally active male adults.

A number of individuals in the present investigation self-selected exercise intensities that resulted in a low and, sometimes, negative affective response. Certain physiological factors may result in negative affective responses that are not related to the ventilatory breakpoint. For example, individuals with decreased thermoregulatory ability resulting in higher core and brain temperatures may experience lower pleasure during exercise (Maw, Boutcher \& Taylor, 1993). In addition, individuals who experience skeletal and joint aches and pains may experience 
decreased pleasure during exercise (Ekkekakis \& Lind, 2006). However, the current investigation did not measure these potential underlying factors.

\subsection{RECOMMENDATIONS FOR FUTURE RESEARCH}

The affective response to exercise must be studied using longitudinal models to show whether FS-R is indeed related to exercise adherence. These models could test the response of affect to exercise training and, potentially, changes in physical fitness. Longitudinal studies of selfselected exercise intensity, which may be associated with a positive affective response, could demonstrate how self-selected exercise intensity changes over time and with changes in fitness level. In addition, the relation of the affective response with physical activity level is unknown. Valid measurements of physical activity, such as accelerometry, should be used to compare individuals of low and high physical activity. For example, subjects' physical activity levels could be measured over a period of time for the classification into a low or high physical activity group prior to the assessment of self-selected exercise intensity. Future studies should include the affective response to acute exercise trials, both constant-intensity and intervals, as well as short-term and long-term exercise programs using self-selected and imposed exercise intensities. The exercise sessions studied should include those designed to maximize psychological and/or physiological benefits.

Future research should also explore the underlying factors that influence self-selected exercise intensity. The current investigation studied the affective response to self-selected exercise in recreationally active subjects who self-reported participation in 50 to 150 minutes of 
aerobic physical activity per week. Physical activity status measured using accelerometry should be employed to obtain valid estimations of weekly energy expenditure to compare subjects with low and high levels of physical activity.

Future research could include the development of an exercise discomfort index (EDI) that includes measurements of RPE, ratings of muscle pain (RMP) specific to the exercise stimulus, and the affective response (FS-R). The following equation could be used: EDI $=$ RPE + RMP FS-R. The EDI would increase with an increase in RPE, an increase in RMP, and a decrease in FS-R. The EDI of subjects could be compared during self-selected and imposed exercise intensities. For example, a subject performs a submaximal, self-selected exercise session equivalent to the ventilatory breakpoint. His average RPE is 5 (using the 0-10 OMNI Scale), his average RMP is 3 (using the 0-10 OMNI Pain Scale), and his average FS-R is +2 (using the Feeling Scale). This exercise session would result in an EDI of $5+3-2=6$. The same subject performs a submaximal, imposed exercise session equivalent to $130 \%$ of the ventilatory breakpoint. His average RPE is 8 , his average RMP is 6 , and his average FS-R $=-3$. This exercise session would result in an EDI of $8+6-(-3)=8+6+3=17$.

Additional future research could investigate the impact of age, gender, clinical status, exercise environment (e.g. laboratory, fitness facility, community center, outdoors), as well as exercise mode or the preference of exercise mode. These future investigations should compare the affective response to self-selected and imposed exercise intensities. In addition, they should compare individuals of low and high fitness. 


\subsection{LIMITATIONS}

One of the primary limitations of the present paradigm is that the exercise conditions were performed in a laboratory and subjects were outfitted with a heart rate monitor and a metabolic mouthpiece throughout each exercise session. This potentially awkward and, most likely, uncomfortable environment is much different than where the subjects may choose to exercise regularly, such as outdoors or at a familiar fitness facility located at school or in the community. Sheppard and Parfitt (2008) noted that the extrapolation of laboratory-based findings to field settings could be questionable. In addition, Dishman and Buckworth (2002) stated that the affective response to exercise in the laboratory environment could be quite different than that experienced in a self-selected, more familiar environment. Future research should compare the affective response to a similar physical stimulus in both the laboratory and in various other settings, such as outdoors or at a familiar gym located at a school or in the community.

Lind and colleagues (2008) report than the order of testing may impact the affective response during exercise. Therefore, the affective responses recorded in the present investigation may have been influenced by the sequence of testing. However, since the imposed exercise intensity trial performed during Session 3 was dependent on the exercise intensities selected by the subjects during Session 2, performed one week previous, these sessions could not be counterbalanced. The interval of one week between Sessions 2 and 3 was selected to allow for an ample amount of time for memory fade pertaining to both the physical stimuli and the affective responses during the previous exercise trial. It is possible that one week did not result in the desired memory fade in young, recreationally active adult males. However, from an 
ecological perspective, the separation of Sessions 2 and 3 by only 1 or 2 days may have been more appropriate for the generalizability of the results to the health-fitness setting.

Sheppard and Parfitt (2008) observed that knowledge of exercise duration may have an effect on the affective response to exercise. In the current investigation, subjects were aware the exercise trials were 20 minutes in duration. In addition, as FS-R values were estimated in 5minute intervals, the subjects were also aware of the remaining duration of the exercise trial.

Finally, as stated by Parfitt and colleagues (2000), the Feeling Scale may be too simplistic to describe the affective response to exercise because it has been shown that exercise may have a differential response on positive and negative affect (Lox \& Rudolph, 1994; McAuley \& Courneya, 1994; Rudolph \& Kim, 1996). The Subjective Exercise Experiences Scale has been used in these studies to assess separately both positive and negative affect, as well as fatigue (McAuley \& Courneya, 1994). Another multi-dimensional affect measurement scale is the Exercise-induced Feeling Inventory (Gauvin \& Rejeski, 1983). However, given the body of literature that has utilized the Feeling Scale, it was felt to be an appropriate quantification of affect for the current investigation and will allow for comparison to previous studies.

\subsection{CONCLUSIONS}

The present investigation found that, in young, recreationally active adult males, there was no difference in the affective response to an imposed exercise intensity that was previously selfselected between those who were and were not aware that the intensity was, in fact, the same. One primary reason for this result may be the inter-individual variability in the estimation of 
affect as well as in the change in affect from the self-selected to the imposed exercise condition. However, despite the variability in the affective response, a significant proportion of subjects self-selected exercise intensities above 50\% of oxygen uptake reserve. This intensity is recommended by the American College of Sports Medicine as an important threshold above which the achievement of physiological benefit is likely.

Exercise recommendations for special populations, and for any individual who seems resistant to the adoption of regular exercise, should not only take into account what is recommended from a physiological perspective, but what is seen as tolerable and enjoyable from a psychological perspective (Biddle \& Fox, 1998; Brownell, 1995; Castellani et al., 2003). It has been shown that the amount of time spent during a given situation can depend on the affect experienced during the activity (Emmons \& Diener, 1986). Therefore, the acute affective response to exercise may influence future exercise participation. Exercise perceived as feeling pleasant may lead to future participation. On the other hand, exercise perceived as feeling unpleasant could decrease future participation or lead to withdrawal from the activity altogether (Parfitt et al., 2006). The goal, then, would be to maximize the positive affective response that patients or clients experience during exercise, which may be the link in the chain between exercise and adherence (Wankel, 1993). Perhaps, even in individuals who do not self-select an exercise intensity that produces a physiological overload response leading to cardiorespiratory benefit, the focus should still be to allow these individuals to perform exercise at a preferred level. This may be especially important in previously sedentary individuals attempting to begin an exercise program. Measurable physiological benefits, although ultimately necessary, especially in the overweight and obese, may have to be de-emphasized at the onset of an exercise program. However, this sensitive time period for special populations must be carefully yet 
specifically guided by trained professionals who can motivate these individuals to find the internal drive to continue participation. The exercise professionals should carefully develop a progressive strategy considering both physical and psychological performance during exercise so the patients or clients can ultimately reach levels of intensity that will lead to cardiorespiratory benefits and the maintenance or reduction of body weight. 
APPENDIX A

[FEELING SCALE]

$\begin{array}{ll}+5 & \text { Very Good } \\ +4 & \\ +3 & \text { Good } \\ +2 & \text { Fairly Good } \\ +1 & \text { Neutral } \\ 0 & \text { Fairly Bad } \\ -1 & \\ -2 & \\ -3 & \text { Bad } \\ -4 & \\ -5 & \text { Very Bad }\end{array}$




\section{APPENDIX B}

\section{[FEELING SCALE INSTRUCTIONS]}

Read to subject:

Throughout the exercise session, I will ask you to rate how you feel using the Feeling Scale (point to scale). While participating in exercise, it is quite common to experience changes in how you feel. You may find that exercise can be pleasant (and make you feel good), or you may find it to be unpleasant (and make you feel bad). Additionally, your feelings may change over time. You might feel good and bad a number of times during exercise. The Feeling Scale is used to measure these responses. The scale ranges from negative 5, 'very bad', to positive 5, 'very good'. '0' represents neutral feeling, neither good nor bad. Your answer may stay the same or may change, and there are no incorrect answers. Please point to the number that best describes how you are feeling when you are asked. 
APPENDIX C

\section{[ANOVA TABLES FOR PRIMARY HYPOTHESIS]}

\section{Tests of Within-Subjects Effects}

Measure:MEASURE_1

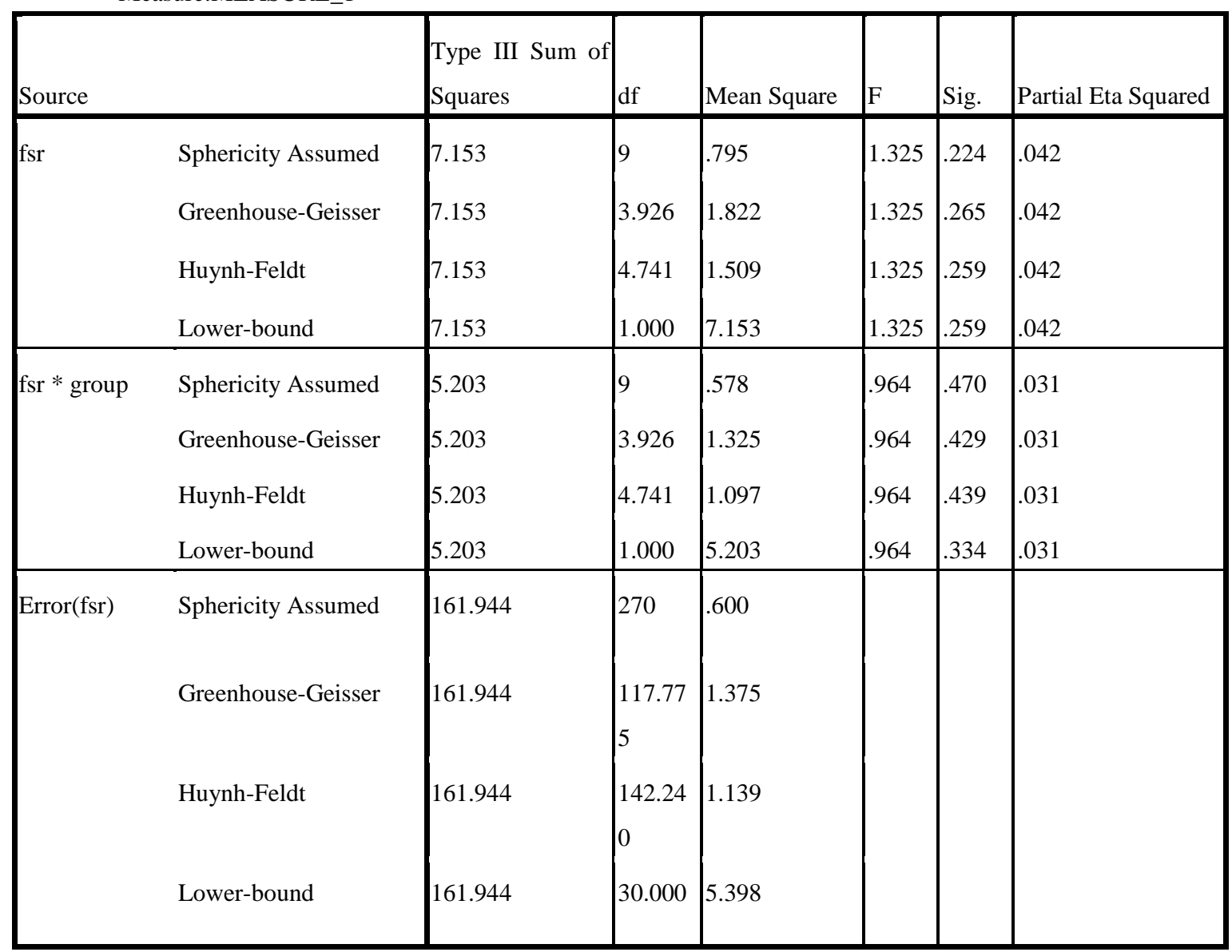


Tests of Between-Subjects Effects

Measure:MEASURE_1

Transformed Variable:Average

\begin{tabular}{|l|r|l|l|l|l|l|}
\hline & \multicolumn{2}{|l|}{ Type III Sum of } & & & & \\
Source & Squares & df & Mean Square & F & Sig. & Partial Eta Squared \\
\hline Intercept & 1.128 & 1 & 1.128 & .277 & .602 & .009 \\
group & 9.453 & 1 & 9.453 & 2.322 & .138 & .072 \\
Error & 122.1 & 30 & 4.071 & & & \\
\hline
\end{tabular}




\section{BIBLIOGRAPHY}

Acevedo, E. O., Kraemer, R. R., Haltom, R. W., and Tryniecki, J. L. (2003). Perceptual responses proximal to the onset of blood lactate accumulation. J Sports Med Phys Fitness, 43, 267-273.

American College of Sports Medicine. (2006). ACSM's guidelines for exercise testing and prescription $\left(7^{\text {th }}\right.$ ed). Philadelphia, PA: Lippincott Williams \& Wilkins.

Biddle, S. J. H., and Fox, K. R. (1998). Motivation for physical activity and weight management. Int J Obes Relat Metab Disord, 22 (Suppl 2), S39-S47.

Bixby, W. R., Spalding, T. W., and Hatfield, B. D. (2001). Temporal dynamics and dimensional specificity of the affective response to exercise of varying intensity: Differing pathways to a common outcome. J Sport Exerc Psychol, 23, 171-190.

Borg, G. (1962). Physical performance and perceived exertion. Lund, Sweden: Gleerup.

Caserta, M. S., and Gillett, P. A. (1998). Older women's feelings about exercise and their adherence to an aerobic regimen over time. Gerontologist, 38, 602-609.

Brownell, K. D. (1995). Exercise and obesity treatment: psychological aspects. Int J Obes Relat Metab Disord, 19 (Suppl 4), S122-S125.

Castellani, W., Ianni, L., Ricca, V., Manucci, E., and Rotella, C. M. (2003). Adherence to structured physical exercise in overweight and obese subjects: a review of psychological models. Eating Weight Disord, 8, 1-11.

Cox, K. L., Burke, V., Gorely, T. J., Beilin, L. J., and Puddey, I. B. (2003). Controlled comparison of retention and adherence in home- vs center-initiated exercise interventions in women ages $40-65$ years: The S.W.E.A.T. Study (Sedentary Women Exercise Adherence Trial). Prev Med, 36, 17-29.

Damasio, A. R. (1995). Toward a neurobiology of emotion and feeling: Operational concepts and hypotheses. Neuroscientist, 1, 19-25.

Davies, R. C., Rowlands, A. V., and Eston, R. G. (2008). The prediction of maximal oxygen uptake from sub-maximal ratings of perceived exertion elicited during the multistage fitness test. Br J Sports Med, 42, 1006-1010, Epub. 
Deci, E. L., \& Ryan, R. M. (1985). Intrinsic motivation and self-determination in human behavior. New York: Plenum Press.

Department of Health and Human Services. (1999). Promoting physical activity: A guide to community action. Champaign, IL: Human Kinetics.

Dishman, R. K., and Buckworth, J. (2002). Exercise Psychology. Human Kinetics: Champaign, IL.

Dishman, R. K., Farquhar, R. P., and Curetone, K. G. (1994). Responses to preferred intensities of exertion in men differing in activity levels. Med Sci Sports Exerc, 26, 783-790.

Ekkekakis, P. (2003). Pleasure and displeasure from the body: Perspectives from exercise. Cogn Emot, 17, 213-239.

Ekkekakis, P., Hall, E. E., and Petruzzello, S. J. (2004). Practical markers of the transition from aerobic to anaerobic metabolism during exercise: Rationale and a case for affect-based exercise prescription. Prev Med, 38, 149-159.

Ekkekakis, P., Hall, E. E., and Petruzzello, S. J. (2005). Variations and homogeneity in affective responses to physical activity of varying intensities: An alternative perspective on doseresponse based on evolutionary considerations. J Sport Sci, 23, 477-500.

Ekkekakis, P., Hall, E. E., Van Lunduyt, L. M., \& Petruzzello, S. J. (2000). Walking in (affective) circles. Can short walks enhance affect? J Behav Med, 23, 245-275.

Ekkekakis, P., and Lind, E. (2006). Exercise does not feel the same when you are overweight: the impact of self-selected and imposed intensity on affect and exertion. Int J Obes, 30, 652-660.

Ekkekakis, P., and Petruzzello, S. J. (1999). Acute aerobic exercise and affect: current status, problems and prospects regarding dose-response. Sports Med, 28, 337-374.

Ekkekakis, P., \& Petruzzello, S. J. (2002). Analysis of the affect measurement conundrum in exercise psychology: IV. A conceptual case for the affect circumplex. Psychol Sport Exerc, 3, 35-63.

Emmons, R.A., and Diener, E. (1986). A goal-affect analysis of everyday situational choices. $J$ Res Pers, 20, 309-326.

Eston, R. G., Lambrick, D., Sheppard, K., and Parfitt, G. (2008). Prediction of maximal oxygen uptake in sedentary males from a perceptually regulated, sub-maximal graded exercise test. J Sports Sci, 26, 131-139.

Frijda, N. H. (1988). The laws of emotion. Am Psychol, 43, 349-358. 
Glass, S. C., \& Chvala, A. M. (2001). Preferred exertion across three common modes of exercise training. J Strength Cond Res, 15, 474-479.

Godin, G. (1987). Importance of the emotional aspect of attitude to predict intention. Psychol Rep, 61, 719-723.

Godin, G., Desharnais, R., Valois, P., and Bradet, R. (1995). Combining behavioral and motivational dimensions to identify and characterize the stages in the process of adherence to exercise. Psychol Health, 10, 333-344.

Goosey-Tolfrey, V., Lenton, J., Goddard, J., Oldfield, V., Tolfrey, K., and Eston, R. (2010). Regulating intensity using perceived exertion in spinal cord-injured participants. Med Sci Sports Exerc, 42, 608-613.

Hall, E. E., Ekkekakis, P., and Petruzzello, S. P. (2002). The affective beneficence of vigorous exercise revisited. Br J Health Psychol, 7, 47-66.

Hardy, C. J., \& Rejeski, W. J. (1989). Not what but how one feels: The measurement of affect during exercise. J Sport Exerc Psychol, 11, 304-317.

Kang, J., Hoffman, J. R., Walker, H., Chaloupka, E. C., and Utter, A. C. (2003). Regulating intensity using perceived exertion during extended exercise periods. Eur J Appl Physiol, $89,475-482$.

Kang, J., Chaloupka, E. C., Biren, G. B., Mastrangelo, M. A., and Hoffman, J. R. (2009). Regulating intensity using perceived exertion: Effect of exercise duration. Eur J Appl Physiol, 105, 445-451.

Kirkcaldy, B. C., \& Shephard, R. J. (1990). Therapeutic implications of exercise. J Sport Psychol, 21, 165-184.

Laaksonen, D. E., Niskanen, L. K., Lakka, H., Rauramaa, R., Salonen, J. T., and Lakka, T. A. (2002). Low levels of leisure-time physical activity and cardiorespiratory fitness predict development of the metabolic syndrome. Diabetes Care, 25, 1612-1618.

Lee, J. Y., Jensen, B. E., Oberman, A., Fletcher, G. F., Fletcher, B. J., and Raczynski, J. M. (1996). Adherence in the training levels comparison trial. Med Sci Sports Exerc, 28, 4752.

Lind, E., Ekkekakis, P., \& Vazou, S. (2008). The affective impact of exercise intensity that slightly exceeds the preferred level: 'Pain' for no additional 'gain.' J Health Psychol, 13(4), 464-468.

Lind, E., Joens-Matre, R. R., and Ekkekakis, P. (2005). What intensity of physical activity do previously sedentary middle-aged women select? Evidence of a coherent pattern from physiological, perceptual, and affective markers. Prev Med, 40, 407-419. 
Maw, G. J., Boutcher, S. H., and Taylor, N. A. S. (1993). Ratings of perceived exertion and affect in hot and cool environments. Eur J Appl Physiol, 67, 174-179.

Moses, J., Steptoe, A., Mathews, A., \& Edwards, S. (1989). The effects of exercise training on mental well-being in the normal population: A controlled trial. J Psychosom Res, 33, 4761.

Omar, R., \& McAuley, E. (1993). Intrinsic motivation and exercise behavior. J Health Educ, 24, 232-238.

Parfitt, G., Rose, E. A., \& Burgess, W. M. (2006). The psychological and physiological responses of sedentary individuals to prescribed and preferred intensity exercise. $\mathrm{Br} J$ Health Psychol, 11, 39-53.

Parfitt, G., Rose, E. A., \& Markland, D. (2000). The effect of prescribed and preferred intensity exercise on psychological affect and the influence of baseline measures of affect. $J$ Health Psychol, 5(2), 231-240.

Pate, R. R., Blair, S. N., Haskell, W. L., et al. (1995). Physical activity and public health: A recommendation from the Center for Disease Control and Prevention and the American College of Sports Medicine. JAMA, 273, 402-407.

Perri, M. G., Anton, S. D., Durning, P. E., et al. (2002). Adherence to exercise prescriptions: Effects of prescribing moderate versus higher levels of intensity and frequency. Health Psychol, 21, 452-458.

Prasad, D. S., and Das, B. C. (2009). Physical inactivity: A cardiovascular risk factor. Indian J of Med Sci, 63(1), 33-42.

Rejeski, W. J., Best, D., Griffith, P., and Kenney, E. (1987). Sex-role orientation and the responses of men to exercise stress. Res $Q, 58,260-264$.

Robertson, R. J., Goss, F. L., Dube, J., Rutkowski, J., Dupain, M., Brennan, C., and Andreacci, J. (2004). Validation of the adult OMNI scale of perceived exertion for cycle ergometer exercise. Med Sci Sports Exerc, 36, 102-108.

Rose, E. A., and Parfitt, G. (2007). A quantitative analysis and qualitative explanation of the individual differences in affective responses to prescribed and self-selected exercise intensities. J Sport Exerc Psychol, 29, 281-309.

Ryan, R. M., Frederick, C. M., Lepes, D., Rubio, N., and Sheldon, K. M. (1997). Intrinsic motivation and exercise adherence. Int J Sport Psychol, 28, 335-354.

Ryan, R. M., and Deci, E. L. (2000). Self-determination theory and the facilitation of intrinsic motivation, social development, and well-being. Am Psychol, 55, 68-78. 
Sheppard, K. E., and Parfitt, G. (2008). Acute affective responses to prescribed and self-selected exercise intensities in young adolescent boys and girls. Pediatr Exerc Sci, 20, 129-141.

Steptoe, A., \& Bolton, J. (1988). The short-term influence of high and low intensity exercise on mood. Psychol Health, 2, 91-106.

Tate, A. K., \& Petruzzello, S. J. (1995). Varying the intensity of acute exercise: Implications for changes in affect. J Sports Med Phys Fitness, 35, 295-302.

Van Lunduyt, L. M., Ekkekakis, P., Hall, E. E., and Petruzzello, S. J. (2000). Throwing the mountains into the lakes: On the perils of nomothetic conceptions of the exercise-affect relationship. J Sport Exerc Psychol, 24, 151-169.

Venables, M. C., Jeukendrup, A. E. (2009). Physical inactivity and obesity: Links with insulin resistance and type 2 diabetes mellitus. Diabetes/Metab Res Rev, 25 Suppl 1, S18-S23.

Wankel, L. M. (1993). The importance of enjoyment to adherence and psychological benefits from physical activity. Int J Sports Psychol, 24, 151-169.

Yeung, R. R. (1996). Acute effects of exercise on mood. J Psychosom Res, 40, 123-141. 